\title{
Smart Health Internet of Thing for Continuous Glucose Monitoring: A Survey
}

\author{
Nuhu Yusuf ${ }^{*}$, Aminu Hamza ${ }^{1}$, Rilwan Sabo Muhammad ${ }^{1}$, Maryam Aminu \\ Suleiman $^{1}$ and Zainab Aminu Abubakar ${ }^{1}$
}

\author{
${ }^{1}$ Abubakar Tafawa Balewa University Bauchi, \\ Bauchi, Bauchi State, 234, NIGERIA \\ *Corresponding Author
}

DOI: https://doi.org/10.30880/ijie. 2020.12.07.006

Received 26 February 2020; Accepted 3 August 2020; Available online 30 August 2020

\begin{abstract}
Health monitoring system allows patients to monitor the health-related problem to avoid further complications which could result in loss of life. Smart health is one of the categories of a health monitoring system that uses Smartphone's and sensors to effectively monitor patient health status. However, the smart health internet of thing methods for glucose monitoring still does not provide accurate glucose reading. Hence, diabetes patient can easily loss life. To help understand this challenge, a comprehensive survey focused on smart health internet of thing methods for continuous glucose monitoring was conducted. The paper discusses the benefit and challenge of each method applicable to glucose monitoring. It was observed that several smart health methods required sensor to function. Smart vehicles and remote monitoring have less attention. However, when accommodates can provide future opportunities.
\end{abstract}

Keywords: Smart health, internet of things, continuous glucose monitoring, health monitoring system, diabetics monitoring

\section{Introduction}

Glucose monitoring is essentially an important part of diabetes treatment which can avoid further health complication. Consequently, monitoring glucose for diabetes patient captured the attention of many researchers such as Vettretti et al [1], Alfian et al [2], Rigla et al [3], Cheng et al [4], Ye et al [5] and Gia et al [6]. These researchers concentrate mainly on using the application to monitor glucose in diabetes-related problems.

Generally, the existing glucose monitoring categorized based on either devices or technologies. Devices detect glucose level to improve the health status of a diabetes patient. Compared with devices, the technologies provide a connection between the devices and users to ensure that the glucose levels are maintained within the acceptable ranges.

Continuous glucose monitoring [1] is widely used among other glucose monitoring to provide real-time health status response of diabetes patient. Consequently, many continuous glucose monitoring methods have been proposed for monitoring diabetes patient. Gia et al [6] presented real-time monitoring and detection of glucose through signals. Rigla et al [3] described some of the artificial intelligence techniques used in monitoring glucose levels of diabetes patients. More recently, Steck et al [7] investigated the importance of continuous glucose monitoring for children with diabetes so that any type I diabetes issues can be detected as early as possible. Also, they examine $23 \mathrm{Ab}+$ that are at high risk of diabetes settings.

Glucose monitoring based on smart health IoT contained a sensor that can measure the levels of glucose. The alarm alerted either the medical personnel or patient relatives when the glucose level is higher or lower. However, glucose monitoring still difficult to achieve proper monitoring of diabetes patients as their techniques needs to be adjusted to include some important features. Firstly, heart rate, especially faster heart rate has a direct connection to the high risk of diabetes and it may likely affect the accuracy of monitoring diabetes patient. This is because patients with 
diabetes have a chance of getting heart diseases such as heart attack. Secondly, many glucose monitoring methods consider smart sensors to detect the glucose level. These depend completely on sensor effectiveness to be able to accurately present glucose reading and may result in poor results, especially if the sensor signal interferes with others. Thirdly, sleep can positively affect the glucose level of a diabetes patient and the present methods are inefficient of alerting diabetes patient to sleep for some hours to avoid high glucose level.

In this paper, we provide an overview of recent trends and challenges in smart health internet of things for continuous glucose monitoring. The smart health internets of thing are technologies that provide more flexibility with very long communication ranges using smart devices. Other sections of the paper are organized as follows: Section 2 presents background information about continuous glucose monitoring. Section 3 presents a demonstration of the smart health monitoring system. The review of the smart health internet of things for continuous glucose monitoring diabetes methods is presented in section 4. Finally, conclusion and future work are presented in section 5.

\section{Continuous Glucose Monitoring}

Alnahdi and Liu [8] suggested a new mobile IoT model that utilizes hardware, software and stakeholders to improve any change of location for mobile internet of things. Their models can work in different environments including health, especially where places where health facilities are inadequate. One good thing about this model is its ability to get first-hand information from users in terms of feedback for further improvements. Moreover, Dudhe et al [9] briefly examine the application of IoT in monitoring the health conditions of patients. The paper described how patient that is far away from hospitals can extremely benefit from accessing medical attentions from personnel. A sensor device is usually attached to the patient body either in the form watch or belt. The sensor will be synchronized with the patient box by recording patient health status and alert to the doctor via server when the status reached critical conditions that required medical attention. The sensor also communicated to the server via a mobile device which in turn communicates to a medical doctor.

Battelino et al [10] summarize continuous glucose monitoring recommendations for diabetes patients due to its low utilization. Moreover, Taylor et al [11] examine the use of real-time continuous glucose monitoring for type 2 diabetes. The study serves as a preliminary investigation for glucose control. However, Jernevl et al [12] review the benefits and challenges of an optical measurement method for continuous glucose monitoring. This is providing better alternatives for the electrochemical sensor. In addition to that, optical fibre is another platform [13] that could help improve continuous glucose monitoring. They combined smartphone and optical power metre to record glucose signals outputs. Continuous glucose monitoring was also studied in [14] where better results are achieved for free style libre. This can provide better diabetics control measures, specifically in neonatal [15]. Leelarathna et al [16] evaluate glucose control based on continuous glucose monitoring index. Also, hypoglycaemia [17] can be avoided when continuously monitor glucose level. Miller et al [18] present longitudinal changes in CGM taking into consideration Germany and Austrian context.

Raun and Saha [19] present how the lives of several individuals could be saved when adopting smart health system based on IoT devices. The paper described the frequent loss of people lives due to improper monitoring of their health status and suggested a design framework that senses the health status of individual and then alert to hospital in case of emergency. The hospital will then allocate the nearest ambulance to take care of the patient and assign further treatment. In addition to that, Sholla et al [20] examine how ethics can be integrated within smart health IoT applications to deliver efficient services. Social information is one of the important factors for ethics that required careful consideration and inclusion in smart health IoT devices. Therefore, feedbacks on ethics need to be collected and included in the IoT applications.

Lakkis and Elshakankiri [21] focused on highlighting IOT issues in terms of smart health emergency and operations application. Their paper examines six (6) challenges that would make the design of this application possible and suggest the used different technologies to tackle these challenges. Within the proposed solution, the sensor is applied to detect either patient sugar level or blood pressure. Smart health application will be installed on patient Smartphone to detect patient emergency location. The GPS guides ambulances traffic congestion to patient destination and recommend a simple path to reach the destination in time.

Medical smart home significantly improves the health status of patients. Therefore, designing a framework to address the security of patient data moving across the network becomes important. Rauscher and Bauer [22] present a security framework for IoT application in the medical domain. Their work integrates languages so that safety signs would be properly understood. Security vulnerability remains. However, Venkatesh et al [23] suggest a health IOT application design to enhance response time based on the specific problem context. For instance, during an emergency, it can easily identify the health status of patients and their locations so that doctors would be assigned to medical care.

To guarantee the reliability of IoT applications in the health sector, Shaik et al [24] provide survey studies of different proposed solutions to the data management of patients. Radiofrequency identification is an important aspect that connects sensors, patient body system and health ambulances. But issues of privacy have always been reported on the use of RFID technology. The paper also identified named data networking as a security solution for all data movement across devices. In named data networking, the hidden Markov model ensures any health-related changes have been detected. 


\section{Smart Health Monitoring Architectures Categorization}

This section presents smart health monitoring system architectures which glucose in diabetes person can also be part of it. The diabetes patients mean people contacted with type 2 diabetes.

Smart health internet of thing has gained much attention from health care industries and researchers due to its effectiveness in continuous monitoring patient of health status. Many health care organizations and hospitals are now integrating smart health systems into their systems to improve patients' health [25] and save a life.

To monitor patient health status continuously, a smart health IoT device uses different technologies to ensure endto-end communication between the patient and the hospital or health organization.

There are many architectures use in developing smart health but this paper identified the most commonly architecture/frameworks about continuous health monitoring of patient status. These architectures are based on protocols, layers, cloud and sensors. Figure 1 show these architectures classifications

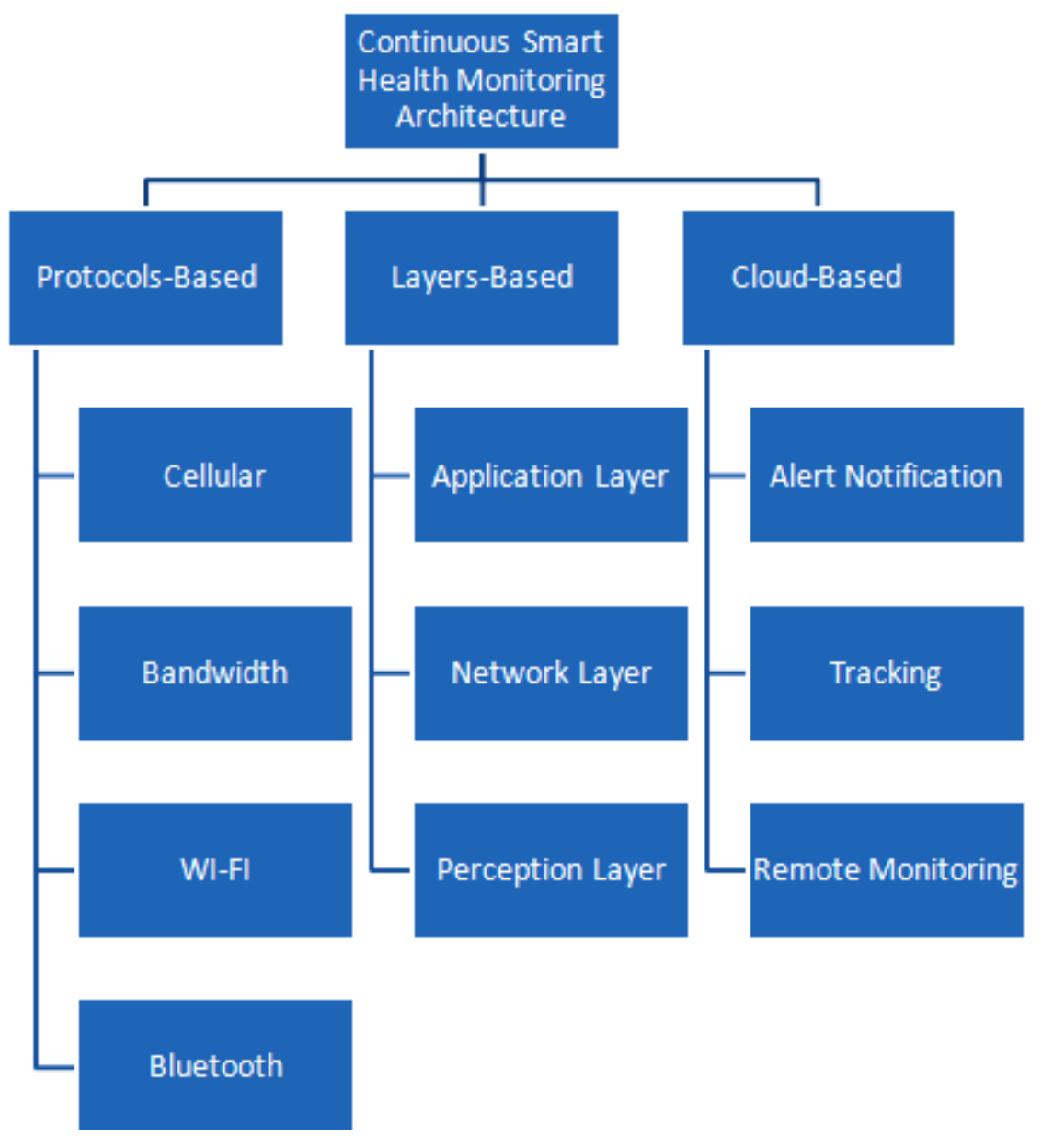

Fig. 1 - cloud-based smart monitoring system

\subsection{Protocol-Based Architectures}

Protocols-based architecture helps in gathering patients' data from various IoT devices attached to a health monitoring system. This architecture considers the wireless network [26] to communicate among devices used with lower power consumption.

Bandwidth architecture can ensure that small packets with data rates communicate faster across devices within seconds, for example, $100 \mathrm{mb} / \mathrm{s}$. In determining the bandwidth consumption per seconds, the average range of successful communication should be taking into consideration for smart health monitoring. Hospitals usually require at least $100 \mathrm{mbps}$ bandwidth to allow real-time medical image and patient data transfers.

Cellular networks can communicate to a wide area environment using SMS. The environment covers many kilometres. The frequently used cellular technologies are 3G, 4G, 5G and LTF. For instance, Houze de I'Aulnoit et al [5] present 4G connection in fatal electronic health care monitoring. Ahad et al [2] present 5G IoT architecture for smart health care where they identified small cells as an essential component for achieving larger miles range. The 
small cells can cover up to 30KM. Moreover, Sigwele et al [1] suggest 5G utilizes gateway via Bluetooth to save energy.

Although cellular network helps in producing efficient architecture, the network traffic [27] and coverage usually varies according to the number of users within the environment. In Wi-Fi protocols-based architecture, several computers and medical devices are connected to each other via a wireless network with authentication protocols[28]. It can connect a device attached to the patient and the hospitals' devices [5]. This will enable doctors to be alerted during emergency cases [4]. Wi-Fi can easily set up at a reasonable cost for better services. Application protocols such as WiFi and cellular networks are very important as they appear in most of the current healthcare IOT architectures [3].

\subsection{Layers-Based Architecture}

Layers-based architectures take into account both the healthcare and patient environments. Once the environment is identified, proper IOT technologies are then applied. Layer-based architectures comprise an application, network and perception layers. These layers can help to provide real-time support for health monitoring [2].

Application layers receive much greater attention as it usually contains user interface parts. According to Onasanya and Elshakankiri [3] cancer care layer which is part of an application layer can be used in conjunction with the data centre layer, hospital layer and security layers to monitor cancer patients.

Another important layer is the network layer which provides security management [29] for data transport across devices. This will ensure various smart health services and devices are served efficiently without compromise. The security measures can be enforced at different network layers [1] to ensure end-to-end patients and service delivery.

Perception layer helps to perceive all physical objects, sensors and actuators around the patients and medical personnel. These will enable gathering enough data that could reveal the patient health status. The data is usually presented to the application layer for decision making.

\subsection{Cloud-Based Architecture}

In cloud-based health architecture, smart health devices with a large data connection can easily be managed for efficient real-time health monitoring. Also, different devices can easily be integrated in cloud structure. Amar et al [1] present a cloud-based mobile health care architecture by providing online patient record access and medical support services with emergency medical service. The heart rate of diabetes patient can be monitor to avoid further health complication issues. According to Hyun and Kyungyong [30], heart rate due to stress can be monitor using the sensor. Due to the presence of the sensor, both higher and lower rate could be easily detected by the sensor. It is also necessary to provide medical supports to the patient in case of emergency. However, during patient exercise, the heart rate will vary depending on the nature of the exercises.

Alert notification is based on feedback action allows easy notification sending medical personnel. These notifications can be in the form of either text, audio or images. The alert notification is needed especially during an emergency to quickly and efficiently communicate to protect patients from worsening health conditions. Alert can be in two ways; one way which sends to a particular medical practitioner and two ways which consider subgroups of medical practitioners. The advantage of two ways is in case one medical personnel is busy then others will quickly respond. To avoid higher glucose level due to the absence of sleep, we use sleep alert to notify the patient on the need to rest to avoid further health complications problems. We assume that only patient will be notified not the doctors. To ensure that the doctors have access to alert records due to absence of sleep, the doctor can log in to patient records to see the details including alerts.

Tracking both patients, ambulances and nearest medical personnel could also be possible with the help of GPS. The GPS provides all information about the patient location and the less busy route available for an ambulance. Tracking ensures real-time monitoring and improves patient health service efficiently. After sleep alerts and the patient unable to sleep which resulted to higher glucose level, the sensor will notify to the ambulance via GPRS/4G network and GPS. The nearest ambulance with fewer traffic connections on the road will be assigned to the patient. We assumed that each ambulance contains medical personnel who will provide a first aid medical assistance before meeting a specialized doctor. Upon receiving the patient, the ambulance will locate the nearest specialized doctor using GPS and then assign a patient to that doctor. After the ambulance assigns a patient to a doctor, the system then alerts the doctor. In this case, the doctor has the right to accept or reject during a busy schedule. In case of rejection, the ambulance will look for another doctor again until getting acceptance.

Although remote monitoring has similarity with tracking structure, the sensors can be embedded on a device in remote monitoring to measure patient health status. These devices range from different diseases. Some diseases require to centralize repository at a particular health care provider which stores sensors data. While others only need local storage of data at patients' home site. For example, Fig. 2 presents a typical cloud-based smart monitoring system. 


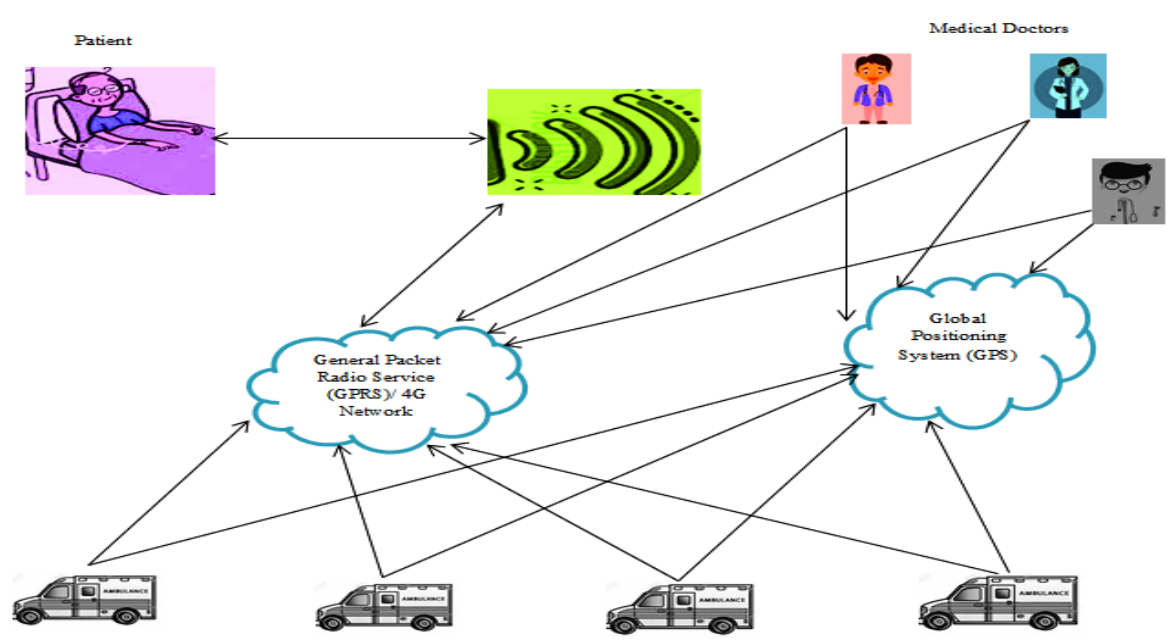

Fig. 2 - cloud-based smart monitoring system

\section{Smart Health IOT for Continuous Glucose Monitoring Methods}

This section presents a comprehensive review of various approaches to smart health internet of things for continuous glucose monitoring. It provides the classification of these approaches taking into consideration their benefits and limitations.

The smart health IOT for continuous glucose monitoring methods can be categorized depending on either supporting the input or output stage of glucose estimate. Figure 3, presents the categorization of these methods. The smart sensor transmits glucose readings over wireless to the receiver. The smartphone serves as the receiving device for monitoring glucose level. Finally, smartwatch serves as an interactive device that functions similar to Smartphone but monitors heart rate and sleep.

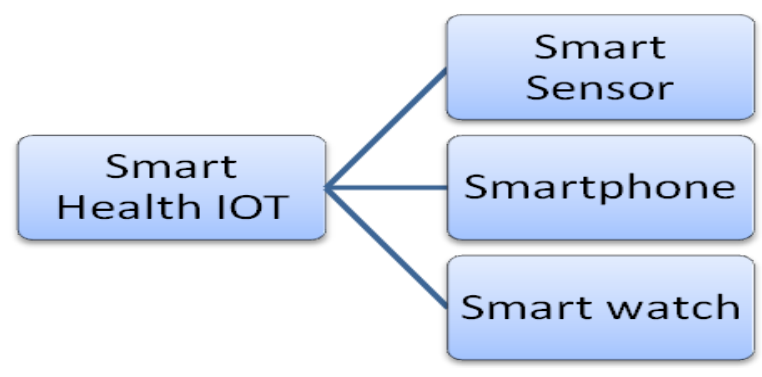

Fig. 3 - smart health IOT for glucose monitoring system

\subsection{Smart Sensor Method}

The smart sensors are techniques for smart health IoT by enhancing sensors to avoid interference with other sensors while continue monitoring glucose level. The smart sensor operates in such a way that specific signal input will detect, process and passing it to the receiver. Table 2 presents some smart sensor methods by taking into consideration their advantages and limitations.

Mahmoud and Moh'd [31] present non-enzymatic graphene-based biosensors to ensure that accurate and reliable glucose reading can be obtained from the sensor. The method shows its capability of enhancing any interference that may come from body contact with fluids. One of the shortcomings of this method is the inability to address interference that may come from other devices.

Vahlsing et al [32] proposed a photoplethysmograph signal-based smart sensor method which obtains sensors from a device attached to a diabetes patient so that glucose level can be continuously monitored to maintain patient health status. The limitation of this method is that it doesn't show directly how glucose reading can be estimated but rather serve as a trial method. Moreover, Aishwarya et al [33] examines other glucose monitoring method s and suggest that optical method can serve as a future improvement if the emphasis is on cost-effective. 


\subsection{Smartphone Method}

These methods are prominently smart health IOT techniques used to continuously monitor glucose at any level and at a particular time. Smartphones contain sensors and capable of supporting wireless communication with additional functionality to monitor diabetes patient glucose level. Jihad et al [34] present a smartphone technique for continuous monitoring not only glucose but also other health-related problems. One of the advantages of this method is the ability to monitor and visualize glucose remotely. Though, it only considers a single Arabian language and captures other languages.

Rahmat et al [35] proposed glucose monitoring IOT-based which can send a glucose reading via mobile phone. Their work tries to address the shortcomings of conventional invasive methods by presenting non-invasive as an alternative. Furthermore, various techniques can be converted into a non-invasive method with monitoring functionality including smartphone in reading diabetes patient glucose level.

\subsection{Smart watch}

As we introduced before, smartwatch works similar to a smartphone but can include sleep monitoring and heart rate. Smartphone proves to be inefficient because most of the diabetes patient has high blood pressure and require normal sleep hours to be able to keep their normal health status. Stradolini et al [36] monitor the health status of diabetes patients while on move. The problem experienced with this method is the sensor interference with other devices. In addition to that, Saravanan [37] present continuous diabetes patient monitoring with heart rate and skin moisture. Smartwatch can easily visualize the glucose level [38]. Kim et al [39] present the impact of smart watch on healthy behavior .

Table 1 - Smart Health Glucose Monitoring Methods

\begin{tabular}{|c|c|c|c|}
\hline Papers & Methods & Advantages & Disadvantages \\
\hline $\begin{array}{l}\text { Mahmoud and } \\
\text { Moh'd [31] }\end{array}$ & Smart Sensor & $\begin{array}{l}\text { Enhanced sensor interference } \\
\text { with body fluids }\end{array}$ & $\begin{array}{l}\text { Some sensors can still } \\
\text { interfere with each } \\
\text { other }\end{array}$ \\
\hline $\begin{array}{l}\text { Vahlsing et al } \\
\text { [32] }\end{array}$ & Smart Sensor & $\begin{array}{l}\text { Photophlythesmograph signal } \\
\text { using a sensor can be } \\
\text { obtained from diabetes } \\
\text { patient }\end{array}$ & It's in the trial stage \\
\hline $\begin{array}{l}\text { Aishwarya et al } \\
\text { [33] }\end{array}$ & Smart Sensor & $\begin{array}{l}\text { Optical method provide } \\
\text { future improvement for cost- } \\
\text { effective }\end{array}$ & \\
\hline Jihad et al [34] & Smart phone & $\begin{array}{l}\text { Can monitor glucose level } \\
\text { remotely and provide } \\
\text { visualization of glucose } \\
\text { reading }\end{array}$ & Language dependent \\
\hline $\begin{array}{l}\text { Rahmat et al } \\
{[35]}\end{array}$ & Smart phone & $\begin{array}{l}\text { Glucose reading can be sent } \\
\text { to a mobile phone }\end{array}$ & \\
\hline Alam et al [40] & Smart phone & $\begin{array}{l}\text { Combine various monitoring } \\
\text { function including SMS }\end{array}$ & Subject to errors \\
\hline $\begin{array}{l}\text { Stradolini et al } \\
\text { [36] }\end{array}$ & Smart watch & $\begin{array}{l}\text { Simultaneous and constantly } \\
\text { monitoring the health status } \\
\text { of the patient while on the } \\
\text { move }\end{array}$ & $\begin{array}{l}\text { Sensor interference } \\
\text { can result in poor } \\
\text { results }\end{array}$ \\
\hline Vashist [38] & Smartwatch & $\begin{array}{l}\text { Provide } \quad \begin{array}{r}\text { continuous } \\
\text { monitoring }\end{array} \text { through } \\
\text { visualizing glucose level }\end{array}$ & Big data challenges \\
\hline Saravanan [37] & Smartwatch & $\begin{array}{l}\text { Provide continuous } \\
\text { monitoring diabetes patients } \\
\text { in which sensor can sense not } \\
\text { only glucose but heart rate, } \\
\text { skin moisture among others }\end{array}$ & $\begin{array}{l}\text { Doctors } \\
\text { included }\end{array}$ \\
\hline
\end{tabular}




\section{Conclusion}

In this paper, a comprehensive review of smart health IoT for glucose monitoring has been presented. It is easy to understand that the IoT technique can be utilized directly in smart health to provide accurate results. Several proposed methods were observed required sensor to function. This is because the sensor is capable of detecting the signal.

Smart vehicles and remote monitoring have less attention. However, when accommodates can provide future opportunities.

\section{Acknowledgement}

This research project has been sponsored by research fund TETFUND, Academic Staff Training and Development (AST \& D) conference attendance, Abubakar Tafawa Balewa University Bauchi Nigeria.

\section{References}

[1] M. Vettoretti, G. Cappon, G. Acciaroli, A. Facchinetti, and G. Sparacino. (2018). Continuous Glucose Monitoring: Current Use in Diabetes Management and Possible Future Applications. Journal of Diabetes Science Technology, 12, 1064-1071

[2] G. Alfian, M. Syafrudin, M. F. Ijaz, M. A. Syaekhoni, N. L. Fitriyani, and J. Rhee. (2018). A personalized healthcare monitoring system for diabetic patients by utilizing BLE-based sensors and real-time data processing. Sensors (Switzerland), 18, 7

[3] M. Rigla, G. García-Sáez, B. Pons, and M. E. Hernando. (2018). Artificial Intelligence Methodologies and Their Application to Diabetes. Journal of Diabetes Science and Technology, 12, 303-310

[4] A. Cheng, V. Raghavaraju, J. Kanugo, Y. P. Handrianto, and Y. Shang. (2018). Development and evaluation of a healthy coping voice interface application using the Google home for elderly patients with type 2 diabetes. In 15th IEEE Annual Consumer Communications and Networking Conference, 1-5

[5] Q. Ye, U. Khan, S. A. Boren, E. J. Simoes, and M. S. Kim. (2018). An Analysis of Diabetes Mobile Applications Features Compared to AADE7 ${ }^{\mathrm{TM}}$ : Addressing Self-Management Behaviors in People With Diabetes. Journal of Diabetes Science and Technology, 12, 808-816

[6] T. Nguyen Gia et al. (2019). Energy efficient fog-assisted IoT system for monitoring diabetic patients with cardiovascular disease. Future Generation Computer System, 93, 198-211

[7] A. STECK et al. (2018). Continuous Glucose Monitoring Predicts Progression to Diabetes in AutoantibodyPositive Children. Diabetes, 67, 1690

[8] A. Alnahdi and S. H. Liu. (2017). Mobile internet of things (MIoT) and its applications for smart environments: A positional overview. 2017 IEEE 2nd International Congress on Internet of Things, ICIOT 2017, 151-154

[9] P. V. Dudhe, N. V. Kadam, R. M. Hushangabade, and M. S. Deshmukh. (2017). Internet of Things (IOT): An overview and its applications. 2017 International Conference on Energy, Communication, Data Analytics and Soft Computing, ICECDS 2017, 2650-2653

[10] T. Battelino et al. (2019). Clinical Targets for Continuous Glucose Monitoring Data Interpretation: Recommendations From the International Consensus on Time in Range, 42, 1593-1603

[11] P. J. Taylor and C. H. T. N. D. Luscombe-marsh. (2019). Efficacy of Real-Time Continuous Glucose Monitoring to Improve Effects of a Prescriptive Lifestyle Intervention in Type 2 Diabetes : A Pilot Study. Diabetes Therapy, 10, 509-522

[12] I. L. Jernelv, K. Milenko, S. S. Fuglerud, D. R. Hjelme, and A. Aksnes. (2018). A Review of Optical Methods for Continuous Glucose Monitoring. Applied Spectoscopy Review, 54

[13] M. Elsherif, M. U. Hassan, A. K. Yetisen, and H. Butt. (2019). Hydrogel Optical Fibers for Continuous Glucose Monitoring. Biosens. Bioelectron, 137, 25-32

[14] H. Bando et al. (2019). Daily improvement of blood glucose profile by continuous glucose monitoring ( CGM ). Journal of Diabetes, Metabolic Disorders and Control, 6, 24-26

[15] K. Beardsall. (2019). Real time continuous glucose monitoring in neonatal intensive care. Early Human Development, 138, 104844

[16] L. Leelarathna et al. (2019). Evaluating Glucose Control With a Novel Composite Continuous Glucose Monitoring Index. Journal of Diabetes Science and Technology, 1-7

[17] S. Puhr, M. Derdzinski, J. B. Welsh, A. S. Parker, T. Walker, and D. A. Price. (2019). Real-world hypoglycemia avoidance with a continuous glucose monitoring system's predictive low glucose alert. Diabetes Technology and Therapeutics, 21, 155-158

[18] K. M. Miller et al. (2020). Longitudinal changes in continuous glucose monitoring use among individuals with type 1 Diabetes: International comparison in the German and Austrian DPV and U.S. T1D exchange registries. Diabetes Care, 43, E1-E2 
[19] H. N. Saha, N. F. Raun, and M. Saha. (2017). Monitoring patient's health with smart ambulance system using Internet of Things (IOTs). In 2017 8th Industrial Automation and Electromechanical Engineering Conference, 91-95

[20] S. Sholla, R. Naaz, and M. A. Chishti. (2017). Incorporating Ethics in Internet of Things (IoT) Enabled Connected Smart Healthcare. In Proceedings - 2017 IEEE 2nd International Conference on Connected Health: Applications, Systems and Engineering Technologies, 262-263

[21] S. I. Lakkis and M. Elshakankiri. (2018). IoT based emergency and operational services in medical care systems. Jt. 13th CTTE 10th C. Conf. Internet Things - Bus. Model. Users, Networks, 1-5

[22] J. Rauscher and B. Bauer. (2018). Safety and security architecture analyses framework for the internet of things of medical devices. 2018 IEEE 20th International Conference on e-Health Networking, Applications and Services, $1-3$

[23] J. Venkatesh, B. Aksanli, C. S. Chan, A. S. Akyurek, and T. S. Rosing. (2018). Modular and Personalized Smart Health Application Design in a Smart City Environment. IEEE Internet Things Journal, 5, 614-623

[24] Y. Shaikh, V. K.Parvati, and S. R. Biradar. (2018). Survey of Smart Healthcare Systems using Internet of Things (IoT). In 2018 International Conference on Communication, Computing and Internet of Things, 6, 508513

[25] S. A. Butt, D. J. Luis, and T. Jamal. (2019). IoT Smart Health Security Threats. International Conference on Computational Science and Its Applications, 26-31

[26] F. Campioni, S. Choudhury, and F. Al. (2019). Scheduling RFID networks in the IoT and smart health era. Journal of Ambient Intelligence Humanized Computing, 10, 4043-4057

[27] P. D. I. Torino, A. A. Abdellatif, A. Mohamed, C. Chiasserini, M. Tlili, and A. Erbad. (2019). Edge Computing For Smart Health : Context-aware Approaches , Opportunities, and Challenges. IEEE Network, 33, 196-203

[28] H. Hamidi. (2019). An approach to develop the smart health using Internet of Things and authentication based on biometric technology. Future Generation Computer Systems, 91, 434-449

[29] M. Baza, A. Salazar, M. Mahmoud, M. Abdallah, and K. Akkaya. (2018). On Sharing Models Instead of Data using Mimic learning for Smart Health Applications. IEEE International Conference on Informatics, IOT, and Enabling Technology, 231-236

[30] H. Yoo and K. Chung. (2017). Heart rate variability based stress index service model using bio-sensor. Cluster Computing, 21, 1139-1149

[31] M. A. Sakr and M. Serry. (2015). Non-enzymatic graphene-based biosensors for continous glucose monitoring. 2015 IEEE SENSORS, 15-18

[32] T. Vahlsing, S. Delbeck, S. Leonhardt, and H. M. Heise. (2018). Noninvasive Monitoring of Blood Glucose Using Color-Coded Photoplethysmographic Images of the Illuminated Fingertip Within the Visible and NearInfrared Range: Opportunities and Questions. Journal of Diabetes Science and Technology, 12, 1169-1177

[33] K. Aishwarya Lakshmi, R. Rashmi, S. Sadanand, C. K. Narayanappa, and N. Sriram. (2018). Studies on relating to monitoring blood glucose levels using non-invasive optical methods. RTEICT 2017 - 2nd IEEE International Conference of Recent Trends Electron. Information Commununication Technology, 2111-2113

[34] A. J. Jihad, S. S. Mathew, S. Paul, and D. P. Pushpalatha. (2017). Continuous health monitoring using smartphones-A case-study for monitoring diabetic patients in UAE. In Proceedings of the 2016 12th International Conference on Innovations in Information Technology, 61-65

[35] M. A. Aizat Rahmat, E. L. M. Su, M. Mohd Addi, and C. F. Yeong. (2017). GluQo: IoT-based non-invasive blood glucose monitoring. Journal of Telecommunication, Electronic and Computer Engineering, 9, 71-75

[36] F. Stradolini, E. Lavalle, G. De Micheli, P. M. Ros, D. Demarchi, and S. Carrara. (2017). Paradigm-Shifting Players for IoT: Smart-Watches for Intensive Care Monitoring. International. Conference on Wireless Mobile Communication Healthcare. Lecture Notes of the Institute for Computer Sciences, Social Informatics and Telecommunications Engineering, 192, 71-78

[37] M. Saravanan and R. Shubha (2018). Non-invasive Analytics Based Smart System for Diabetes Monitoring. Lecture Notes of the Institute for Computer Sciences, Social Informatics and Telecommunications Engineering, $225,88-98$

[38] J. H. Vashist, S. K., \& Luong. (2017). Point-of-care glucose detection for diabetic monitoring and management. CRC Press, 168

[39] M. Kim, L. Chen, and S. Park. (2019). Factors influencing on smart health. International Journalof Industrial Distribution and Business, 10, 17-23

[40] M. M. Alam et al. (2019). D-CARE: A Non-invasive Glucose Measuring Technique for Monitoring Diabetes Patients. Proceeding of International Joint Conference on Computational Intelligence, 443-453 